\title{
Phase II Conjugates of Urolithins Isolated from Human Urine and Potential Role of $\boldsymbol{\beta}$-Glucuronidases in Their Disposition ${ }^{\mathbb{}}$
}

\author{
Jakub P. Piwowarski, Iwona Stanisławska, Sebastian Granica, Joanna Stefańska, \\ and Anna K. Kiss
}

Department of Pharmacognosy and Molecular Basis of Phytotherapy, Faculty of Pharmacy (J.P.P., I.S., S.G., A.K.K.), and Department of Pharmaceutical Microbiology, Centre for Preclinical Research and Technology (CePT) (J.S.), Medical University of Warsaw, Warsaw, Poland; Primary Laboratory of Origin: Medical University of Warsaw, Faculty of Pharmacy

Received January 24, 2017; accepted March 1, 2017

\begin{abstract}
In recent years, many xenobiotics derived from natural products have been shown to undergo extensive metabolism by gut microbiota. Ellagitannins, which are high molecular polyphenols, are metabolized to dibenzo[b,d]pyran-6-one derivatives-urolithins. These compounds, in contrast with their parental compounds, have good bioavailability and are found in plasma and urine at micromolar concentrations. In vivo studies conducted for ellagitannincontaining natural products indicate their beneficial health effects toward inflammation and cancer, which are associated with the formation of urolithins. However, the great majority of in vitro experiments that have revealed the molecular mechanisms responsible for the observed effects were conducted for urolithin aglycones. These studies are thus incongruent with the results of pharmacokinetic studies that clearly indicate that glucuronide conjugates are the dominant metabolites present in plasma,
\end{abstract}

\section{Introduction}

Accumulating evidence has revealed the diverse effects of the gut microbiota on human health, including nutrition, physiology, and host metabolism. In recent years, the metabolic cross-talk between a host and its gut microbiota in modulating the pharmacokinetics and pharmacodynamics of drugs has gained much attention (Klaassen and Cui, 2015; Yip and Chan, 2015). Many xenobiotics derived from food products and medicinal plant materials have been shown to undergo extensive metabolism by gut microbiota, resulting in the formation of small molecular metabolites that not only are more bioavailable than parental compounds but also reveal interesting pharmacological properties (Kieran and Del Rio, 2015). One of the most widely studied gut

The project was financially supported by a Polish Ministry of Science and Higher Education research grant, luventus Plus [Grant IP2015 062274]. J.P.P. was financially supported by a Foundation for Polish Science START scholarship [START 84.2016]. The project was performed using Centre for Preclinical Research and Technology infrastructure financed by the European Regional Development Fund within the "Innovative Economy" Operational Programme for 2007-2013.

https://doi.org/10.1124/dmd.117.075200.

S This article has supplemental material available at dmd.aspetjournals.org. tissue, and urine. The aim of this study was to isolate and structurally characterize urolithin conjugates from the urine of a volunteer who ingested ellagitannin-rich natural products, and to evaluate the potential role of $\beta$-glucuronidase-triggered cleavage in urolithin disposition. Glucuronides of urolithin $A$, iso-urolithin A, and urolithin $B$ were isolated and shown to be cleaved by the $\boldsymbol{\beta}$-glucuronidases released by neutrophils from azurophilic granules upon $\mathbf{N}$-formylmethionine-leucyl-phenylalanine stimulation as well as by Escherichia coli standard strains and clinical isolates from patients with urinary tract infections. These results justify the hypothesis that the selective activation of urolithin glucuronides by $\beta$-glucuronidase, which are present at high concentrations at inflammation and infection sites and in the microenvironments of solid tumors, could locally increase the concentration of bioactive urolithin aglycones.

ABBREVIATIONS: BHI, brain heart infusion; 2D, two-dimensional; ET, ellagitannin; f-MLP, $N$-formylmethionine-leucyl-phenylalanine; GiUA, isourolithin A glucuronide; GUA, urolithin A glucuronide; GUB, urolithin B glucuronide; HBSS, Hanks' balanced salt solution; HPLC, high-performance liquid chromatography; iUA, iso-urolithin A; MUG, 4-methylumbelliferyl- $\beta$-D-glucuronide; UA, urolithin A; UB, urolithin B; UC, urolithin C; UHPLCDAD-MS ${ }^{n}$, ultra-high performance liquid chromatography coupled with diode array detector and ion trap mass spectrometer. 
observed effects (Gonzalez-Sarrias et al., 2009; Piwowarski et al., 2014a, 2015; Gonzalez-Sarrias et al., 2016).

One important issue that has recently been raised is the inconsistency of in vitro bioactivity results compared with in vivo and clinical pharmacokinetic studies. The great majority of in vitro experiments were conducted for urolithin aglycones, making them incongruent with the results of pharmacokinetic studies that clearly indicate that glucuronide conjugates of urolithins are the dominant metabolites present in plasma, tissues, and urine (Tomás-Barberán et al., 2017).

Although urolithin glucuronides are the main metabolites detected in bodily fluids, the highly enzymatically active environment of inflammation and cancer sites should be considered, as they have the potential to trigger structural alterations. One of the most common, abundant enzymes at these sites is $\beta$-glucuronidase, which is released from infiltrating inflammatory cells and the lysosomes of necrotic cells (Tranoy-Opalinski et al., 2014). $\beta$-Glucuronidase is also a characteristic enzyme of Escherichia coli, the bacterial species that is responsible for urinary tract infections, among other disease (Chang et al., 1989).

The aim of the study was to isolate and structurally characterize the urolithin conjugates from the urine of a volunteer after ingestion of ET-rich natural products, and to evaluate whether they undergo cleavage under the influence of $\beta$-glucuronidases released by human neutrophils, as well as by various $E$. coli strains.

\section{Materials and Methods}

Reference Compounds and Chemicals. Standards of urolithin A (UA), isourolithin A (iUA), urolithin B (UB), and urolithin C (UC) were isolated from human gut microbiota cultures using a previously described method (Piwowarski et al., 2016). The purity of examined compounds ( $\geq 95 \%$ ) was confirmed using a ultra-high performance liquid chromatography coupled with diode array detector and ion trap mass spectrometer method. Lythrum salicaria herb aqueous extract was prepared as described previously (Piwowarski and Kiss, 2013). The chromatogram obtained with the UHPLC-DAD-MS ${ }^{\mathrm{n}}$ method, presenting extract's ellagitannin profile, is provided in Supplemental Fig. S2.

Subject and Dosing Procedure. We chose a healthy volunteer (male, age 30) based on our previous study, which identified the volunteer as possessing microbiota that produce the most diverse pattern of urolithins (Piwowarski et al., 2016). The volunteer was asked to supplement his diet with products rich in ETs. Pomegranate juice ( $0.5 \mathrm{l} / \mathrm{day}$ ), walnuts ( $30 \mathrm{~g} / \mathrm{day})$, hazelnuts ( $30 \mathrm{~g} /$ day), and fresh raspberries ( $200 \mathrm{~g} /$ day) were consumed for 5 days. Total urine was collected from day 1 to day 5 and stored at $-20^{\circ} \mathrm{C}$ in a total combined volume of 5.51 of urine. The study respected the 1964 Declaration of Helsinki for the ethical treatment of human subjects for biomedical research

UHPLC Analysis of Urine. An SPE Chromabond $\mathrm{C}_{18}$ Hydra column (Macherey-Nagel, Düren, Germany) was preconditioned with $10 \mathrm{ml}$ of $\mathrm{MeOH}$ and $10 \mathrm{ml}$ of $\mathrm{H}_{2} \mathrm{O}$. Fifteen milliliters of urine was applied to the column and washed with $10 \mathrm{ml}$ of $\mathrm{H}_{2} \mathrm{O}$ before elution with $2 \mathrm{ml}$ of $\mathrm{MeOH}$. The eluate was subjected to UHPLC-DAD-MS ${ }^{\mathrm{n}}$ analysis.

Isolation of Metabolites and Structure Determination. Urine (5.5 1) was concentrated under reduced pressure at $40^{\circ} \mathrm{C}$ to 3.01 and extracted four times with 21 of EtOAc. The aqueous residue (3.01) was subjected to chromatography with a Diaion HP-20 column $(40 \times 5 \mathrm{~cm}$; Supelco, Bellefonte, PA $)$ and subsequently eluted with 3.01 of $\mathrm{H}_{2} \mathrm{O}, 20 \% \mathrm{MeOH}$, and $100 \% \mathrm{MeOH}$. The fraction eluted with $100 \% \mathrm{MeOH}(3.7 \mathrm{~g})$ was chromatographed on a silica gel 60 column $(27 \times 3 \mathrm{~cm})$ eluted with $\mathrm{MeOH}-\mathrm{CHCl}_{3}[0: 100 \rightarrow$ 100:0 (v/v) in 7 steps] to give 104 fractions pooled into 11 main fractions $\left(\mathrm{C}_{1}-\mathrm{C}_{11}\right)$. Fraction $\mathrm{C}_{5}(543 \mathrm{mg})$ was purified using a preparative high-performance liquid chromatography (HPLC) system [LC20-AP instrument (Shimadzu, Kyoto, Japan); Zorbax SB-C $18150 \mathrm{~mm} \times 21.2 \mathrm{~mm} \times 5.0 \mu \mathrm{m}$, $\lambda_{1}=254 \mathrm{~nm}, \lambda_{2}=350 \mathrm{~nm}$, flow $9 \mathrm{ml} / \mathrm{min}$ (Agilent, Santa Clara, CA); mobile phase: $0.1 \% \mathrm{HCOOH}$ in water (A) and $0.1 \% \mathrm{HCOOH}$ in $\mathrm{MeCN}$ (B); elution program: $10-40 \% \mathrm{~B} ; 0-40$ minutes] to give urolithin B glucuronide (GUB) (23.80-24.40 minutes; $25 \mathrm{mg}$ ) and UB (31.5-32.0 minutes; $8 \mathrm{mg}$ ). Fractions $\mathrm{C}_{8}(244 \mathrm{mg})$ and $\mathrm{C}_{9}(37 \mathrm{mg})$ were chromatographed on a Sephadex LH-20 column $(137 \times 2 \mathrm{~cm})$ (Pharmacia Fine Chemicals AB, Uppsala, Sweden), and eluted with a mixture of $\mathrm{MeOH}$ :water $(1: 1, \mathrm{v} / \mathrm{v})$ to obtain 140 fractions pooled into 9 fractions $\left(D_{1}-D_{9}\right)$. Fractions $D_{3}(54 \mathrm{mg})$ and $\mathrm{D}_{4}(16 \mathrm{mg})$ were combined and subjected to a preparative HPLC system (elution program: $15-20 \% \mathrm{~B}$; 0-40 minutes) to give urolithin A glucuronide (GUA) (10.05-12.65 minutes; $31 \mathrm{mg}$ ), iso-urolithin A glucuronide (GiUA) (13.00-14.50 minutes; $6 \mathrm{mg}$ ), and GUB (31.60-33.80 minutes; $4 \mathrm{mg}$ ). Fraction $\mathrm{C}_{7}(1159 \mathrm{mg}$ ) was chromatographed on a Sephadex LH-20 column (MeOH:water, 1:1, v/v) to give 297 fractions pooled into 17 fractions $\left(E_{1}-E_{17}\right)$. Fraction $E_{5}(362 \mathrm{mg})$ was subjected to a preparative HPLC system (parameters the same as for fraction $\mathrm{D}_{3}+\mathrm{D}_{4}$ ) to obtain GUA (10.4-12.55 minutes; $94 \mathrm{mg}$ ), GiUA (12.56-14.5 minutes; $14 \mathrm{mg}$ ), and GUB (31.60-33.80 minutes; $44 \mathrm{mg}$ ). Fractions $\mathrm{D}_{2}(22 \mathrm{mg})$ and $\mathrm{E}_{4}(141 \mathrm{mg})$ were combined and subjected to a preparative HPLC system (parameters the same as for fraction $\mathrm{C}_{5}$ ) to give GUB (23.70-24.50 minutes; $21 \mathrm{mg})$. Fraction $\mathrm{C}_{6}(811 \mathrm{mg})$ was chromatographed on a Sephadex LH-20 column (MeOH:water, 1:1, v/v) to give 241 fractions pooled into 18 fractions $\left(F_{1}-F_{18}\right)$. Fractions $F_{11}(75 \mathrm{mg})$ and $F_{12}(46 \mathrm{mg})$ were combined and subjected to a preparative HPLC system (as for fraction $\mathrm{C}_{5}$ ) to obtain GUB (23.60-24.10 minutes; $45 \mathrm{mg}$ ) and $\mathrm{UB}(31.00-31.70$ minutes; $6 \mathrm{mg})$.

The EtOAc fraction $(2.8 \mathrm{~g})$ was chromatographed on a silica gel 60 column $\left(27 \times 3 \mathrm{~cm}\right.$; Macherey-Nagel) eluted with $\mathrm{MeOH}-\mathrm{CHCl}_{3}[0: 100 \rightarrow 100: 0(\mathrm{v} / \mathrm{v})$ in 9 steps] to give 140 fractions pooled into 10 main fractions $\left(\mathrm{A}_{1}-\mathrm{A}_{10}\right)$. Fractions $\mathrm{A}_{2}+\mathrm{A}_{3}(91.7 \mathrm{mg})$ contained $\mathrm{UB}$ as the major compound, which was purified using preparative HPLC (31.50-32.00 minutes; $4 \mathrm{mg}$ ).

${ }^{1} \mathrm{H}$ and ${ }^{13} \mathrm{C}$ NMR spectra were recorded at $25^{\circ} \mathrm{C}$ on a VARIAN VNMRS instrument (300 MHz for ${ }^{1} \mathrm{H}$ and $75 \mathrm{MHz}$ for ${ }^{13} \mathrm{C} \mathrm{NMR}$ ) (Varian Inc., Palo Alto, CA). Dimethylsulfoxide- $d_{6}$ was used as a solvent. Chemical shifts $(\delta)$ are reported in ppm, and coupling constants $(J)$ are reported in Hz. The assignments were based on heteronuclear single quantum correlation (HSQC) and Heteronuclear Multiple Bond Correlation (HMBC) experiments.

Gut Microbiota Ex Vivo Metabolism. Evaluation of the ex vivo metabolism of ellagitannins by the volunteer's gut microbiota was performed as described previously (Piwowarski et al., 2014b). To acquire anaerobic conditions, a brain heart infusion (BHI; DIFCO, Detroit, MI) was boiled and immediately cooled before the experiment. Forty milligrams of $L$. salicaria $L$. extract (phytochemically standardized ET source) was dissolved in $1 \mathrm{ml}$ of distilled water and sterilized by filtration through an Ophtalsart hydrophilic syringe filter $(0.2 \mathrm{~mm}$; Sartorius Stedim Biotech GmbH, Göttingen, Germany). One milliliter of $10 \%$ fecal slurry in $\mathrm{BHI}$ and $0.5 \mathrm{ml}$ of extract solution were added to $8.5 \mathrm{ml}$ of BHI. The batch cultures were incubated in a sealed container under anaerobic conditions using GENbox anaer sachets (bioMerieux, Marcy-l'Étoile; France) at $37^{\circ} \mathrm{C}$. After 24 hours, the batch culture was extracted three times with $10 \mathrm{ml}$ of diethyl ether. The organic layers were evaporated to dryness and redissolved in $500 \mu \mathrm{l}$ of $\mathrm{MeOH}$ for UHPLC-DAD-MS ${ }^{\mathrm{n}}$ analysis.

Neutrophils Isolation. Peripheral venous blood was taken from four healthy human donors (males, 20-35 years old) at the Warsaw Blood Donation Centre. Donors did not smoke or take any medication. They were clinically confirmed to be healthy, and routine laboratory tests showed values within the normal range. The study conformed to the principles of the Declaration of Helsinki. Neutrophils were isolated with a standard method of dextran sedimentation and subsequent hypotonic lysis of erythrocytes and centrifugation in a Ficoll Hypaque gradient (PAN-biotech, Aidenbach, Germany) (Piwowarski et al., 2014a). The purity of neutrophils was $>97 \%$, and viability as measured by trypan blue exclusion was $>98 \%$.

Deglucuronidation assay. After isolation, neutrophils were resuspended in Hanks' balanced salt solution (HBSS) $\left(6 \times 10^{5} \mathrm{cells} / \mathrm{ml}\right)$ primed with cytochalasin B $(5 \mu \mathrm{g} / \mathrm{ml})$ for 5 minutes and stimulated with $N$-formylmethionine-leucylphenylalanine (f-MLP) $(1 \mu \mathrm{M})$ for 10 minutes. The neutrophils were centrifuged (2000 rpm; 10 minutes; $4^{\circ} \mathrm{C}$ ). The supernatant was acidified with lactic acid to $\mathrm{pH}$ $=5.2$ and incubated with GiUA, GUA, and GUB at a final concentration of $50 \mu \mathrm{M}$. After 24 hours, $300 \mu \mathrm{l}$ of reaction mixture was mixed with $400 \mu \mathrm{l} \mathrm{MeOH}$, subjected to an ultrasonication for 5 minutes and centrifuged $(14,000 \mathrm{~g}$, 10 minutes). The hydrolysis of glucuronides was monitored using the UHPLCDAD-MS ${ }^{\mathrm{n}}$ method. The amount of released $\beta$-glucuronidase in supernatants was determined using a human $\beta$-glucuronidase enzyme-linked immunosorbent assay kit (Wuhan Fine Biologic Technology Co., Ltd, Wuhan, China) according to the manufacturer's instructions.

Deglucuronidation in E. coli In Vitro Cultures. E. coli NCTC 10538 and ATCC 25922 strains and clinical isolates $(\mathrm{C} 1-\mathrm{C} 7)$ were streaked over a Trypticase Soy Agar plate (Becton Dickinson, Franklin Lakes, NJ) and incubated 
for 24 hours at $37^{\circ} \mathrm{C}$. A single colony was used to inoculate $10 \mathrm{ml}$ of $\mathrm{BHI}$, which was incubated for 24 hours at $37^{\circ} \mathrm{C}$. Sixty microliters of the respective glucuronide solution in phosphate-buffered saline was added to $240 \mu \mathrm{l}$ of culture (final concentration of glucuronide: $50 \mu \mathrm{M}$ ) and incubated for 24 hours at $37^{\circ} \mathrm{C}$. Next, $400 \mu \mathrm{l}$ of $\mathrm{MeOH}$ was added to $250 \mu \mathrm{l}$ of culture, subjected to ultrasonication, and centrifuged $(14,000 \mathrm{~g}, 10$ minutes). The hydrolysis of glucuronides was monitored using a UHPLC-DAD-MS ${ }^{\mathrm{n}}$ method.

Chromatographic Methods. UHPLC-DAD-MS ${ }^{\mathrm{n}}$ analyses were performed using a UHPLC-3000 RS system (Dionex, Sunnyvale, CA) with DAD detection and an AmaZon SL mass spectrometer with an Electrospray ionization (ESI) interface (Bruker Daltonik $\mathrm{GmbH}$, Bremen, Germany). A Zorbax SB-C $\mathrm{C}_{18}$ column $(150 \mathrm{~mm} \times 2.1 \mathrm{~mm} \times 1.9 \mu \mathrm{m}$; Agilent, Santa Clara, CA) was used. The mobile phase consisted of $0.1 \% \mathrm{HCOOH}$ in water (A) and $0.1 \% \mathrm{HCOOH}$ in $\mathrm{MeCN}(\mathrm{B})$. The gradient was as follows: $0-5$ minutes $0 \% \mathrm{~B}, 5-15$ minutes $0-10 \% \mathrm{~B}, 15-$ 25 minutes $10-20 \%$ B, 25-35 minutes $20-30 \%$ B, 35-45 minutes $30-50 \%$ B, 4550 minutes $50-100 \% \mathrm{~B}$, and $50-60$ minutes $100 \% \mathrm{~B}$. The column temperature was maintained at $25^{\circ} \mathrm{C}$, and the flow rate was $0.200 \mathrm{ml} / \mathrm{min}$. The eluate was introduced into the ESI interface without splitting, and compounds were analyzed in the negative and positive ion modes with the following settings: nebulizer pressure of $40 \mathrm{psi}$, drying gas flow rate of $9 \mathrm{l} / \mathrm{min}$, nitrogen gas temperature of $300^{\circ} \mathrm{C}$, and capillary voltage of $4.5 \mathrm{kV}$. The mass scan ranged from 100 to $2200 \mathrm{~m} / \mathrm{z}$. UV spectra were recorded from 200 to $400 \mathrm{~nm}$. The presence of UA, iUA, UB, and UC was confirmed by comparing retention times and UV and $\mathrm{m} / z$ spectra with authentic samples. Other compounds were tentatively assigned based on their chromatographic properties-namely, UV and MS spectra.

Statistical Analysis. The results are shown as the mean values \pm S.E.M. The statistical significance of differences between means was determined by one-way analysis of variance. For comparison of results between the groups, Tukey's post hoc test was used. Results with $p$ values $<0.05$ were considered statistically significant. All analyses were performed using Statistica 10 software (Statistica, Tulsa, OK)

\section{Results}

Profile of Urinary Metabolites. UHPLC-DAD-MSn analysis has shown that the regular weekly intake of ET-rich products results in secretion of phase II conjugates of urolithins in urine (Fig. 1) (Fig. 2A). Metabolites were identified based on their retention times and UV and tandem mass spectrometry data. The major metabolite was GUA, which gave a pseudomolecular ion at $\mathrm{m} / z=403$ and an ion fragment at 227, representing a neutral loss of 176 atomic mass units (amu) that is characteristic of glucuronic acid. GiUA, with a similar pseudomolecular ion and fragmentation pattern, was distinguished from GUA based on UV spectra (Gonzalez-Barrio et al., 2011). GUB gave a pseudomolecular ion at $m / z=387$ and a fragment ion at 211. Small amounts of urolithin A sulfate-glucuronide were detected at $\mathrm{m} / z=483$ and the $\mathrm{MS}^{2}$ ion at 307 (neutral loss of $176 \mathrm{amu}$ ), which fragmented to 227 (characteristic for neutral sulfate loss of $80 \mathrm{amu}$ ). Apart from phase II conjugates, small amounts of iUA, UA, and UB were also detected.

Metabolite Isolation and Structural Determination. By means of liquid-liquid extraction, column chromatography, and preparative highperformance liquid chromatography, three major glucuronide metabolites were isolated from human urine-GiUA, GUA, and GUB (Fig. 1) - as was UB. The structures were determined using ${ }^{1} \mathrm{H},{ }^{13} \mathrm{C} \mathrm{NMR}$, HSQC, and HMBC spectra (Table 1). All metabolites were identified as $\beta$-anomers based on the anomeric H1 signal appearing at $\delta 5.0$ as a doublet, with a coupling constant near $7 \mathrm{~Hz}$ (Pearson et al., 2005). The downfield shift of $\mathrm{H} 2$ and $\mathrm{H} 4$ in the GiUA spectrum, compared with its aglycone (Piwowarski et al., 2016), indicates glucuronidation of the $\mathrm{OH}$ group at $\mathrm{C} 3$. The analogous shifts were observed for $\mathrm{H} 2$ and $\mathrm{H} 4$ of urolithin A 3-O-glucuronide and $\mathrm{H} 7$ and $\mathrm{H} 9$ of urolithin A 8-Oglucuronide. The total amounts of obtained compounds were $20 \mathrm{mg}$ of GiUA, $125 \mathrm{mg}$ of GUA, $139 \mathrm{mg}$ of GUB, and $18 \mathrm{mg}$ of UB. GUA was isolated as a mixture of urolithin A-3-O-glucuronide and urolithin A-8$O$-glucuronide as revealed by NMR spectra, which showed two sets of proton (Fig. 3) and carbon signals. It was impossible to separate these two isomers using available chromatographic methods. However, complete proton and carbon assignments were performed based on two-dimensional (2D) NMR spectra (Table 1).

Gut Microbiota Ex Vivo Metabolism. In all, iUA, UA, UB, and UC were determined in the volunteer's gut microbiota cultures (Fig. 2B). Their identities were confirmed by comparison of retention times, UV, and MS data with standard substances. The most abundant peak was observed for iUA, whereas only small amounts of UA were detected.

Deglucuronidation by Human Neutrophils. Neutrophils isolated from human peripheral venous blood were primed with cytochalasin B and subsequently stimulated with f-MLP to release the azurophilic granules containing $\beta$-glucuronidase. The supernatant $\mathrm{pH}$ was adjusted to 5.2, which is known to be an optimal value for enzyme activity (Ho, 1985). Incubation of GiUA, GUA, and GUB with supernatants resulted in enzymatic cleavage of $\beta$-glycosidic bonds and release of the respective aglycones (Figs. $4 \mathrm{~A}$ and 5). The deglucuronidation process was significantly enhanced when neutrophils were stimulated with cytochalasin B and f-MLP in comparison with untreated cells. When $\mathrm{pH}$ was adjusted beyond the optimal values $(\mathrm{pH}=7.2$ in the case of HBSS without lactic acid and $\mathrm{pH}=3.5$ when excessive acidification was applied), deglucuronidation was not observed (data not shown). To evaluate whether $\beta$-glucuronidase is responsible for the cleavage, its concentration was determined using an enzyme-linked immunosorbent assay. Significantly higher enzyme protein concentrations in supernatants were observed upon stimulation. In addition, differences in the amounts

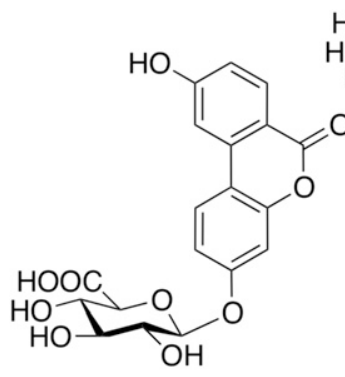

iso-urolithin A

3-O-glucuronide<smiles>O=C(O)CCOc1cc2c(cc1O)oc(=O)c1cc(O)ccc12</smiles>

urolithin A 8-O-glucuronide

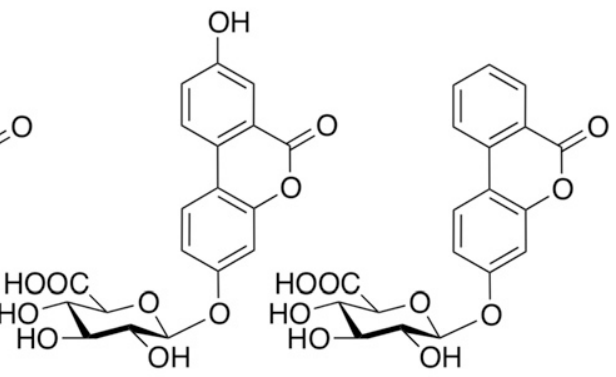

urolithin A 3-O-glucuronide urolithin B

3-O-glucuronide
Fig. 1. Chemical structures of isolated urolithin phase II metabolites. 


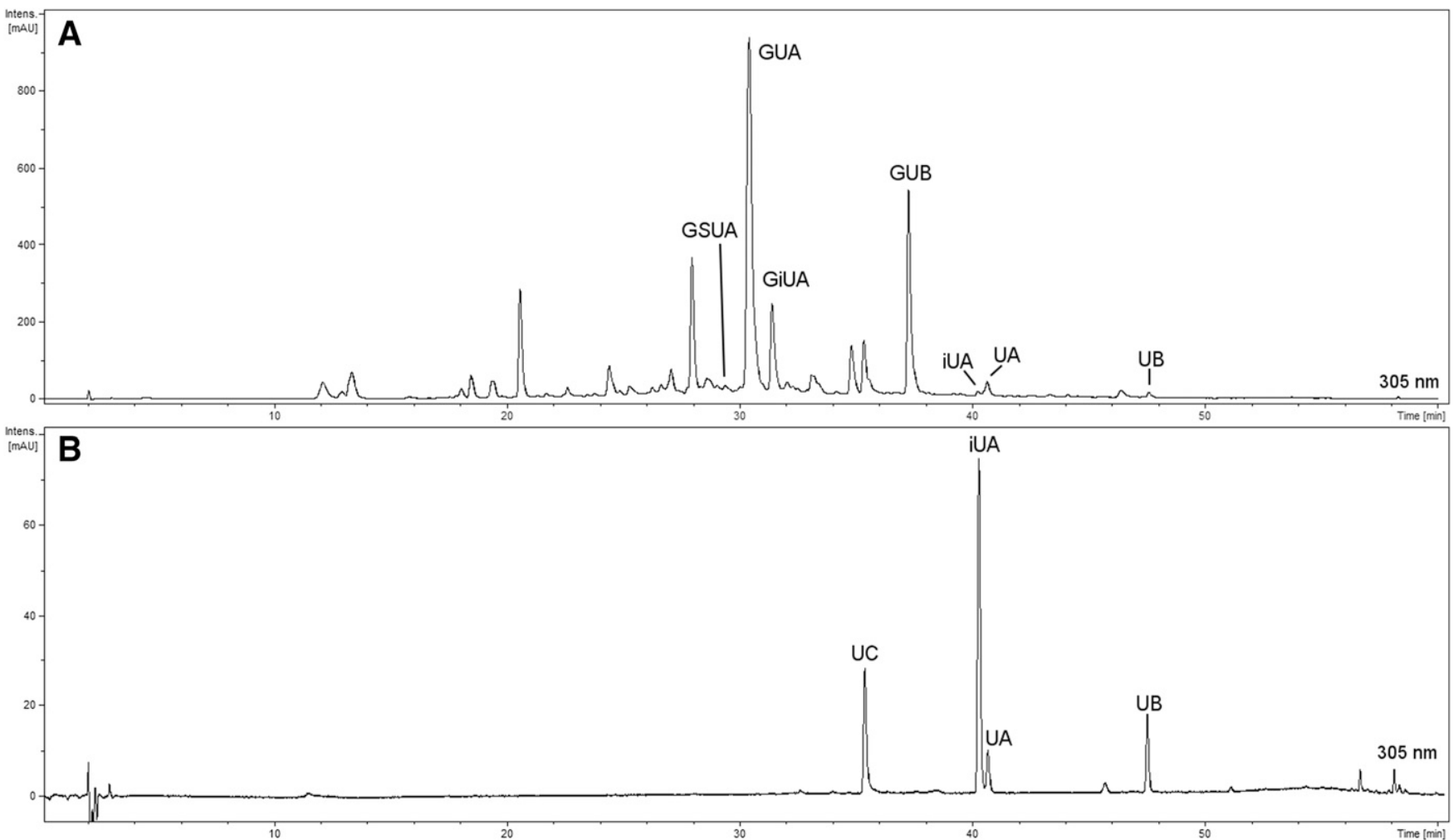

Fig. 2. (A) UHPLC profile of volunteer's urinary metabolites. (B) UHPLC profile of metabolites produced in volunteer's gut microbiota ex vivo culture.

of f-MLP-triggered enzyme release were observed between blood donors (D1-D4) that were strictly correlated with the extent of deglucuronidation (Fig. 4B). No significant differences in the compounds' susceptibility to enzymatic cleavage were observed.

Deglucuronidation in $\boldsymbol{E}$. coli Cultures. Two reference $E$. coli strains, NCTC 10538 and ATCC 25922, and seven clinical isolates (C1C7) obtained from patients suffering from urinary tract infections were used for examinations. Cultures in Fluorocult VRB Agar plates (Merck,
Kenilworth, $\mathrm{NJ}$ ) revealed that the strains expressed different abilities to hydrolyze 4-methylumbelliferyl- $\beta$-D-glucuronide (MUG) (Fig. 6). The most intense blue fluorescence from free 4-methylumbelliferone was observed in the E. coli $\mathrm{C} 1$ strain, whereas E. coli NCTC 10538 and C6 showed no fluorescence. Incubation of GiUA, GUA, and GUB with selected E. coli strains and isolates resulted in deglucuronidation and release of the respective aglycones (Fig. 7; Supplemental Fig. S1). No hydrolysis was observed when compounds were incubated in BHI

TABLE 1

NMR spectroscopic data (300 MHz, dimethylsulfoxide- $d_{6}$ ) for GiUA, GUA, and GUB

\begin{tabular}{|c|c|c|c|c|c|c|c|c|}
\hline & \multicolumn{2}{|c|}{ Iso-urolithin A 3-O-glucuronide } & \multicolumn{2}{|c|}{ Urolithin A 8-O-glucuronide } & \multicolumn{2}{|c|}{ Urolithin A 3-O-glucuronide } & \multicolumn{2}{|c|}{ Urolithin B 3-O-glucuronide } \\
\hline & $\delta_{\mathrm{H}}$ & $\delta_{\mathrm{C}}$ & $\delta_{\mathrm{H}}$ & $\delta_{\mathrm{C}}$ & $\delta_{\mathrm{H}}$ & $\delta_{\mathrm{C}}$ & $\delta_{\mathrm{H}}$ & $\delta_{\mathrm{C}}$ \\
\hline 1 & $8.12(\mathrm{~d}, J=8.9 \mathrm{~Hz})$ & 125.14 & $8.09(\mathrm{~d}, J=8.9 \mathrm{~Hz})$ & 124.37 & $8.15(\mathrm{~d}, J=9.1 \mathrm{~Hz})$ & 123.67 & $8.17(\mathrm{o})$ & 125.14 \\
\hline 2 & $7.05(\mathrm{o})$ & 113.83 & $6.83(\mathrm{dd}, J=8.7,2.4 \mathrm{~Hz})$ & 113.16 & $7.03(\mathrm{dd}, J=8.8,2.5 \mathrm{~Hz})$ & 113.64 & $7.04(\mathrm{dd}, J=8.6,2.1 \mathrm{~Hz})$ & 114.24 \\
\hline 3 & & 159.05 & $10.24(\mathrm{~s})(\mathrm{OH})$ & 159.20 & & 157.39 & & 158.79 \\
\hline 4 & $7.07(\mathrm{o})$ & 104.29 & $6.75(\mathrm{~d}, J=2.4 \mathrm{~Hz})$ & 102.89 & $7.08(\mathrm{~d}, J=2.4 \mathrm{~Hz})$ & 103.86 & $7.03(\mathrm{~s})$ & 104.24 \\
\hline $4 a$ & & 152.57 & & 151.37 & & 150.55 & & 151.96 \\
\hline 6 & & 160.61 & & 160.35 & & 160.39 & & 161.24 \\
\hline $6 a$ & & 112.42 & & 120.08 & & 120.77 & & 119.58 \\
\hline 7 & $8.08(\mathrm{~d}, J=8.7 \mathrm{~Hz})$ & 132.97 & $7.74(\mathrm{~d}, J=2.7 \mathrm{~Hz})$ & 114.90 & $7.54(\mathrm{~d}, J=2.6 \mathrm{~Hz})$ & 113.64 & $8.15(\mathrm{o})$ & 130.16 \\
\hline 8 & $7.02(\mathrm{o})$ & 117.60 & & 156.15 & $10.31(\mathrm{~s})(\mathrm{OH})$ & 157.54 & $7.58(\mathrm{t}, J=7.7 \mathrm{~Hz})$ & 129.05 \\
\hline 9 & & 164.30 & $7.56(\mathrm{dd}, J=8.6,2.7 \mathrm{~Hz})$ & 124.95 & $7.34(\mathrm{dd}, J=8.7,2.7 \mathrm{~Hz})$ & 124.10 & $7.87(\mathrm{t}, J=7.7 \mathrm{~Hz})$ & 136.10 \\
\hline 10 & $7.53(\mathrm{~d}, J=2.1 \mathrm{~Hz})$ & 107.23 & $8.23(\mathrm{~d}, J=9.0 \mathrm{~Hz})$ & 123.64 & $8.18(\mathrm{~d}, J=9.1 \mathrm{~Hz})$ & 123.76 & $8.21(\mathrm{~d}, J=8.9 \mathrm{~Hz})$ & 122.39 \\
\hline $10 \mathrm{a}$ & & 137.28 & & 129.77 & & 126.26 & & 134.70 \\
\hline $10 \mathrm{~b}$ & & 111.52 & & 109.36 & & 112.46 & & 112.46 \\
\hline Glur1 & $5.23(\mathrm{~d}, J=7.2 \mathrm{~Hz})$ & 99.97 & $5.22(\mathrm{~d}, J=7.2 \mathrm{~Hz})$ & 100.25 & $5.21(\mathrm{~d}, J=7.3 \mathrm{~Hz})$ & 99.60 & $5.15(\mathrm{~d}, J=6.8 \mathrm{~Hz})$ & 99.88 \\
\hline Glur2 & $3.32(\mathrm{o})$ & 73.32 & $3.30-3.45(\mathrm{o})$ & 72.99 & $3.30-3.45(\mathrm{o})$ & 72.87 & $3.34(\mathrm{o})$ & 73.14 \\
\hline Glur3 & $3.35(0)$ & 76.18 & $3.30-3.45(\mathrm{o})$ & 75.74 & $3.30-3.45(\mathrm{o})$ & 75.54 & $3.38(\mathrm{o})$ & 75.88 \\
\hline Glur4 & $3.42(\mathrm{o})$ & 71.76 & $3.30-3.45(\mathrm{o})$ & 71.32 & $3.30-3.45(\mathrm{o})$ & 71.29 & $3.40(\mathrm{o})$ & 71.69 \\
\hline Glur5 & $4.03(\mathrm{~d}, J=9.2 \mathrm{~Hz})$ & 75.79 & $3.99(\mathrm{~d}, J=9.2 \mathrm{~Hz})$ & 75.46 & $4.02(\mathrm{~d}, J=9.2 \mathrm{~Hz})$ & 75.34 & $4.02(\mathrm{~d}, J=9.2 \mathrm{~Hz})$ & 75.56 \\
\hline Glur6 & & 170.56 & & 170.05 & & 170.08 & & 170.71 \\
\hline
\end{tabular}

d, doublet; dd, doublet of doublets; o, overlapped signal; s, singlet; t, triplet. 

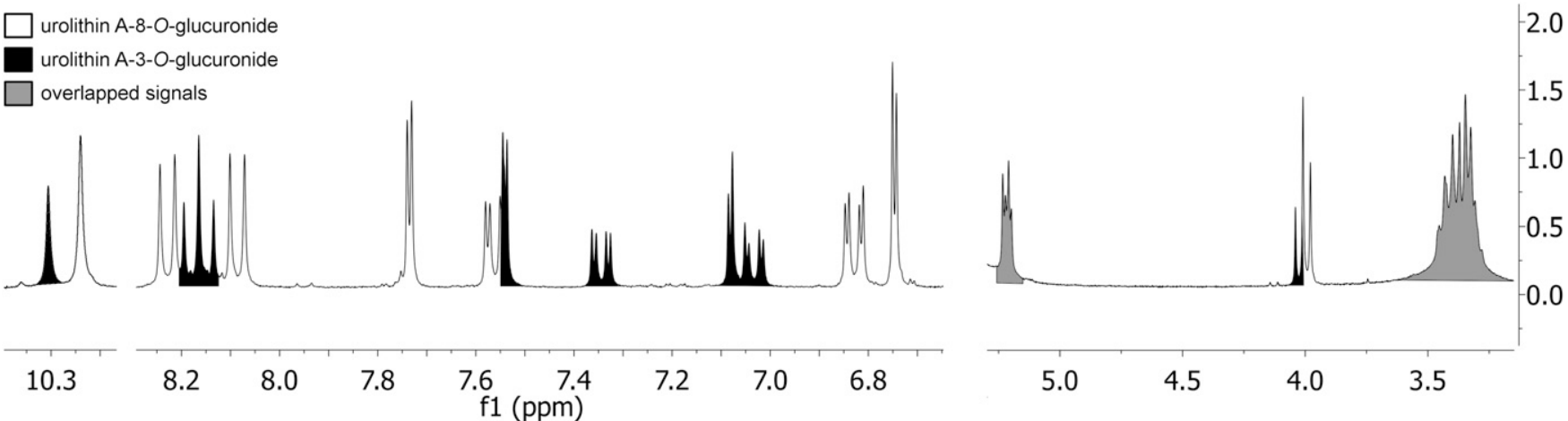

Fig. 3. ${ }^{1} \mathrm{H}$ NMR spectra of urolithin A-3-O-glucuronide and urolithin A-8-O-glucuronide mixture (GUA).

medium without bacteria inoculation. The most active strain was $\mathrm{C} 1$, which was also the most potent in MUG cleavage. The non-MUGdegrading $E$. coli isolate C6 only hydrolyzed the tested glucuronides to a small extent. Comparison of cleavage rates for each glucuronide revealed that, in the case of clinical isolates, GUB was the most susceptible for degradation.

\section{Discussion}

The chosen volunteer was assigned as metabotype B (producing iUA, UA, and UB) (Tomas-Barberan et al., 2014). In addition to phase II metabolites, the respective aglycones were also detected in urine. They were found not only when total collected urine was analyzed (Fig. 2A), but also when samples were analyzed immediately after collection (data not shown), which excludes the possibility of their formation through storage-triggered hydrolysis. The most abundant metabolite was GUA, whereas ex vivo cultures of the volunteer's gut microbiota showed the reverse pattern, with significant dominance of iUA. This observed iUA dominance was in concordance with previous studies conducted on single ETs using the volunteer's gut microbiota [see volunteer D3 in our previous paper (Piwowarski et al., 2016)]. This discrepancy could be explained by different metabolic activity in gut microbiota in vivo and ex vivo or by differences in the metabolic and/or elimination pathways between UA and iUA. Although the volunteer's microbiota were able to produce UC, no phase II metabolites of this compound were detected in urine.

Phase II metabolites of urolithins were isolated from human urine, and their structures were fully described based on the NMR spectra. NMR data for GUA and GUB were in concordance with ${ }^{1} \mathrm{H}$ spectra for compounds obtained through chemical synthesis (Lucas et al., 2009; Gonzalez-Sarrias et al., 2013). The one-dimensional and 2D NMR spectra allowed full assignment of the ${ }^{1} \mathrm{H}$ and ${ }^{13} \mathrm{C}$ signals for the first time. The formation of two GUA regioisomers, i.e., urolithin A 3-Oglucuronide and urolithin A-8-O-glucuronide, was thus fully confirmed in vivo. As in previously described methods for GUA biosynthesis, we were unable to separate the two GUA isomers (Gimenez-Bastida et al., 2012) because they coeluted in the applied column chromatography resins and the analytical RP18 UHPLC column. However, using 2D NMR experiments, we were able to distinguish two series of signals. Based on comparison of their intensities, it can be postulated that glucuronidation at position 8 is favorable. In the future, methods to separate these compounds should be developed. However, this mixture can still be used in bioassays, as it potentially reflects the actual proportion of these metabolites in bodily fluids. The identification presented here is in concordance with previous studies using HPLCTime-of-flight mass spectrometry-solid phase extraction-NMR analysis of urine from patients who ingested tea (van der Hooft et al., 2012). For the first time, GiUA, which was found only as the 3-O-glucuronide, was isolated from human urine. The developed method yields significant amounts of phase II metabolites of urolithins, which then can be applied
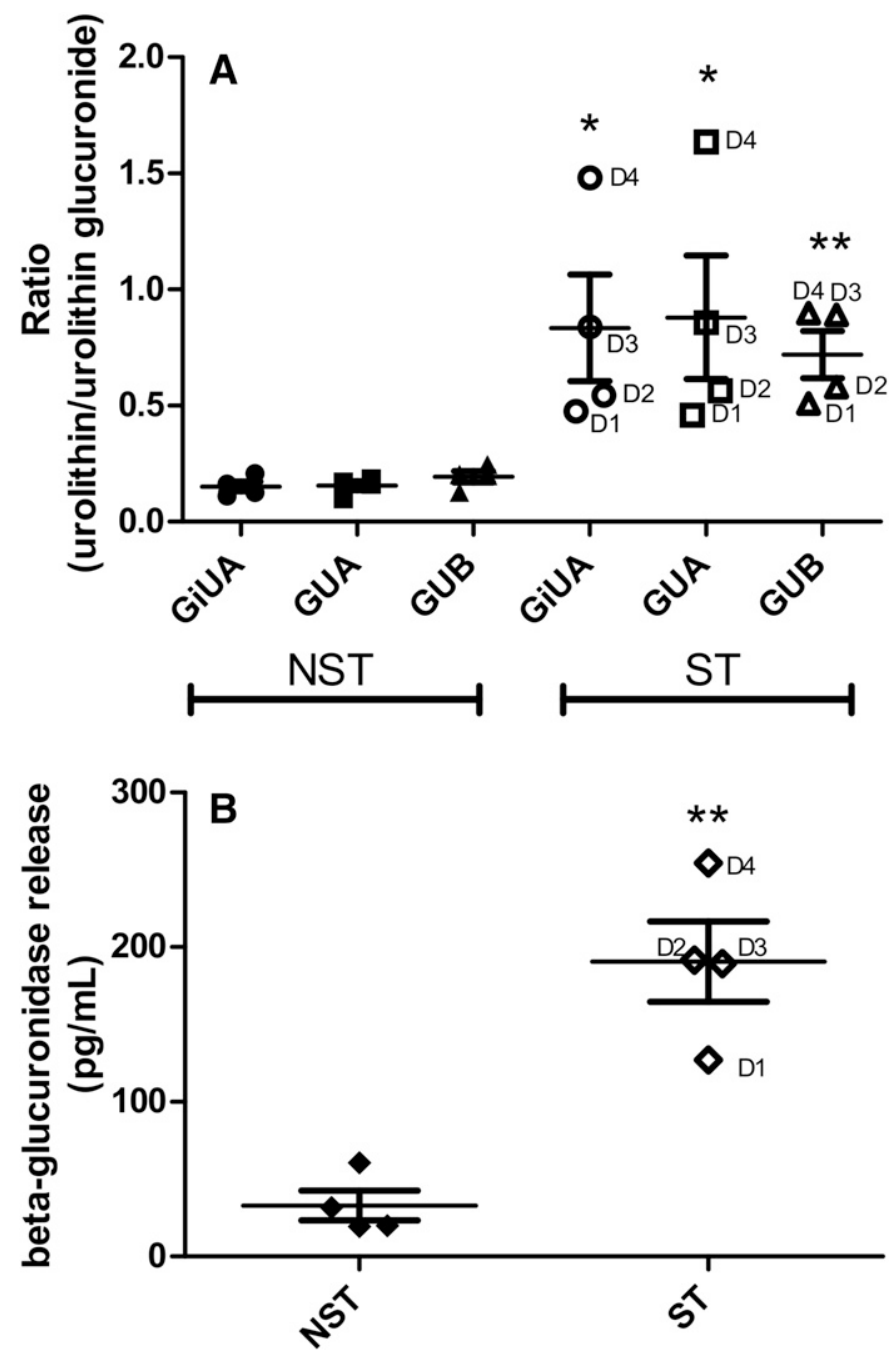

Fig. 4. (A) Cleavage of GiUA, GUA, and GUB by $\beta$-glucuronidase released by human neutrophils obtained from four donors (D1-D4) and assayed in duplicate. (B) Comparison of release of $\beta$-glucuronidase protein between f-MLP-stimulated (ST) and nonstimulated (NST) neutrophils. Statistical significance: $* p<0.05$, $* * p<$ 0.01 versus respective nonstimulated controls. 


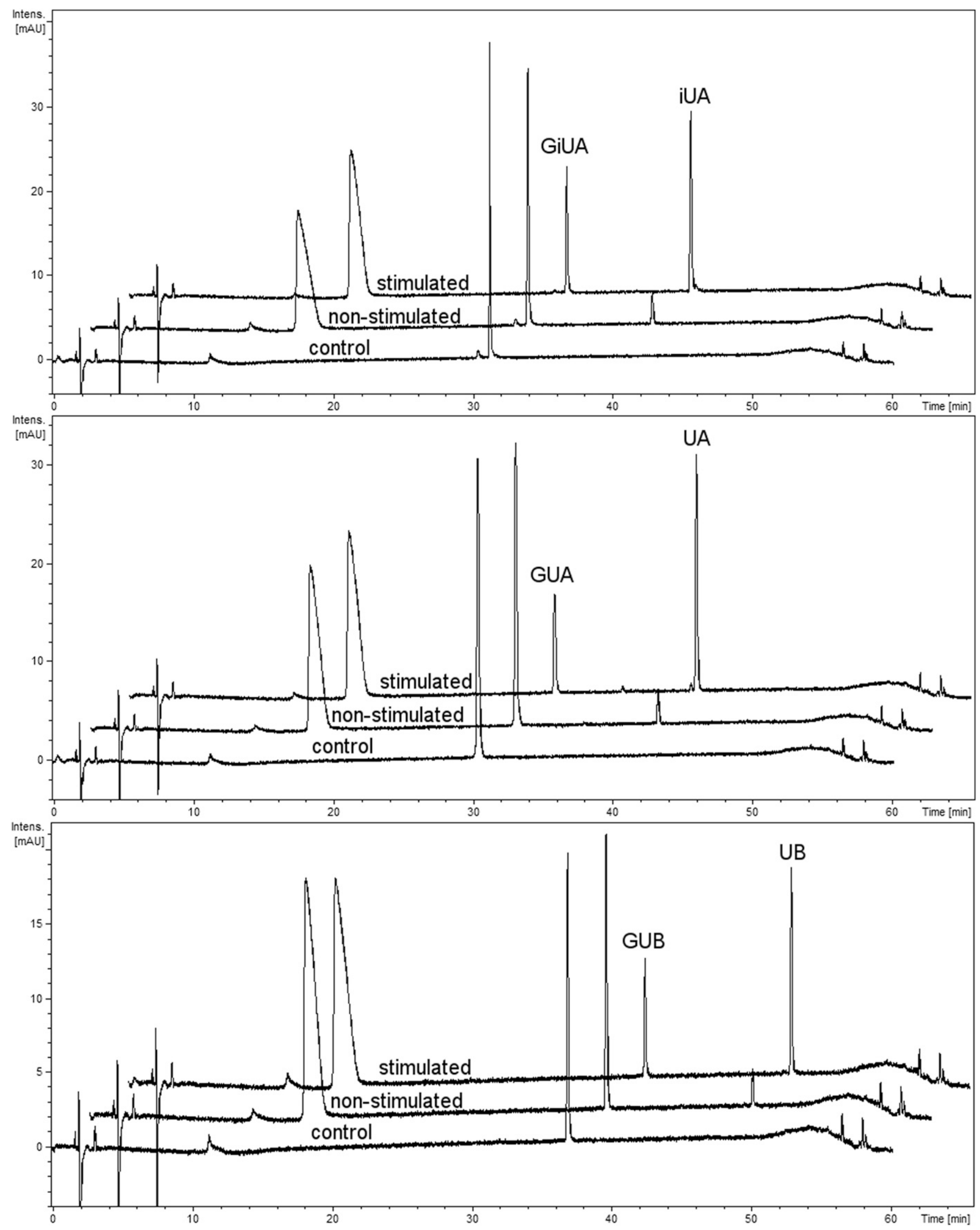

Fig. 5. Chromatograms presenting cleavage of GiUA, GUA, and GUB in acidified supernatants $(\mathrm{pH}=5.2)$ obtained from neutrophils isolated from D4 stimulated with f-MLP. 


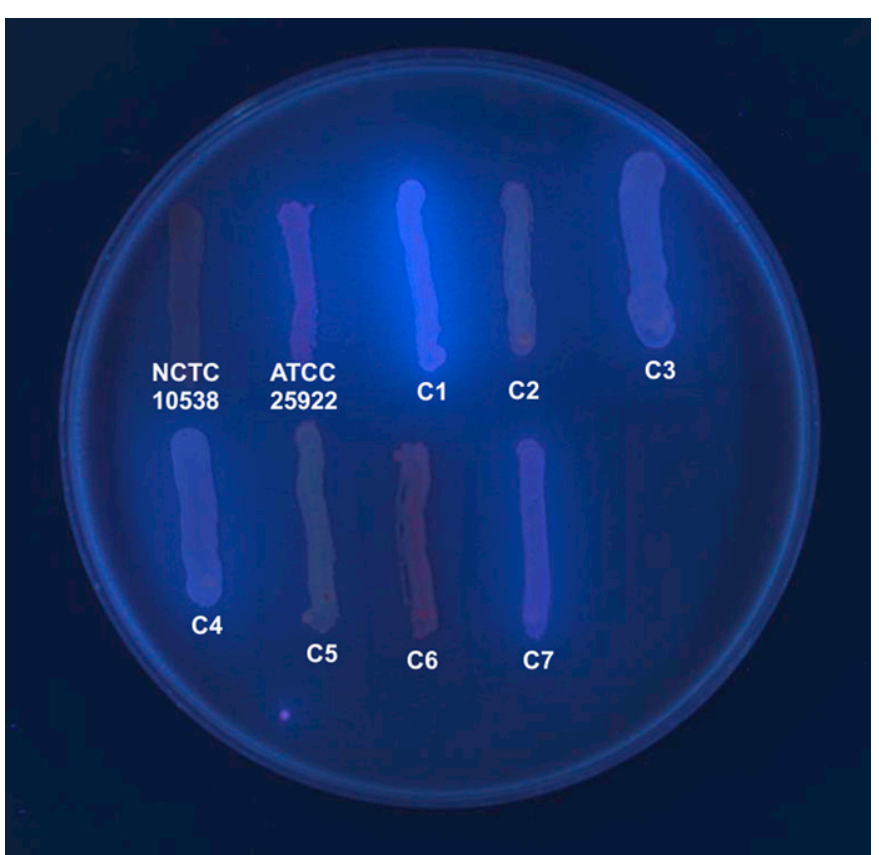

Fig. 6. Cultures of tested E. coli standard strains and clinical isolates (C1-C7) in Fluorocult VRB Agar plates visualized with UV light at $366 \mathrm{~nm}$.

to evaluate the in vivo effects and mechanisms of ET microbiota metabolites. The isolation of GiUA is of particular importance, as 10 $50 \%$ of the population has been identified as iUA producers (TomasBarberan et al., 2014). This compound has not previously been synthesized or isolated from biologic fluids.

The concentration of phase II urolithin metabolites in human plasma and urine can reach 35 and $100 \mu \mathrm{M}$, respectively, whereas aglycones are present in significantly lower concentrations -0.005 and $10 \mu \mathrm{M}$, respectively (Tomás-Barberán et al., 2017). In prostate and colon tissues, urolithin glucuronides were found at nanomolar concentrations (Gonzalez-Sarrias et al., 2010). Both the aglycones and their conjugates ranged from 4.8 and $507.3 \mathrm{ng} / \mathrm{g}$ in human colon tissue samples (NunezSanchez et al., 2014). The administration of UA to rats showed that the distribution of this compound and its conjugated metabolites among different organs reached concentrations up to $1050 \mathrm{ng} / \mathrm{g}$ (Seeram et al., 2007).

Although urolithin conjugates were clearly shown to dominate in plasma, tissues, and urine following the administration of ET-rich natural products, the majority of in vitro studies have focused on nonconjugated molecules. Only a few studies have investigated urolithin phase II metabolites, the majority of which have shown significant in vitro and in silico decreases in activity for conjugated compounds. The phase II metabolism of urolithins in different cell lines is considered a mechanism of cancer cell resistance against urolithins, due to their conversion to glucuronide conjugates, which were shown to exert significantly lower antiproliferative activity (Larrosa et al., 2006; Adams et al., 2010; Dellafiora et al., 2013; Gonzalez-Sarrias et al., 2014; Sala et al., 2015; González-Sarrías et al., 2017). This is the first report of a reversed process of deglucuronidation. Differences in anti-inflammatory activity between GUA and UA have only been shown by GimenezBastida et al. (2012), whereas urolithin methylation was shown to lead to significant activity loss or decrease (Piwowarski et al., 2014a).

In vitro and in vivo studies clearly underline the two major activities of urolithins: anti-inflammatory and antineoplastic (Tomás-Barberán et al., 2017). Additionally, urolithin A was recently shown to improve mitochondrial and muscle function due to the enhancement of mitophagy (Ryu et al., 2016). Nonetheless, low plasma and tissue concentrations of nonconjugated urolithins caused by intensive phase II metabolism render the activities determined for aglycones in vitro inconsistent with in vivo data.

Numerous pathologic conditions, including inflammatory processes and tumors, are known to be associated with significantly increased levels of extracellular $\beta$-glucuronidase. Human $\beta$-glucuronidase is a lysosomal enzyme that accumulates at inflammation sites and in tumor microenvironments due to release from infiltrating neutrophils and macrophages as well as necrotic cells (Tranoy-Opalinski et al., 2014). Mitochondrial respiration defects observed in inflamed and neoplastic tissues lead to aerobic glycolysis—also known as the Warburg effect-resulting

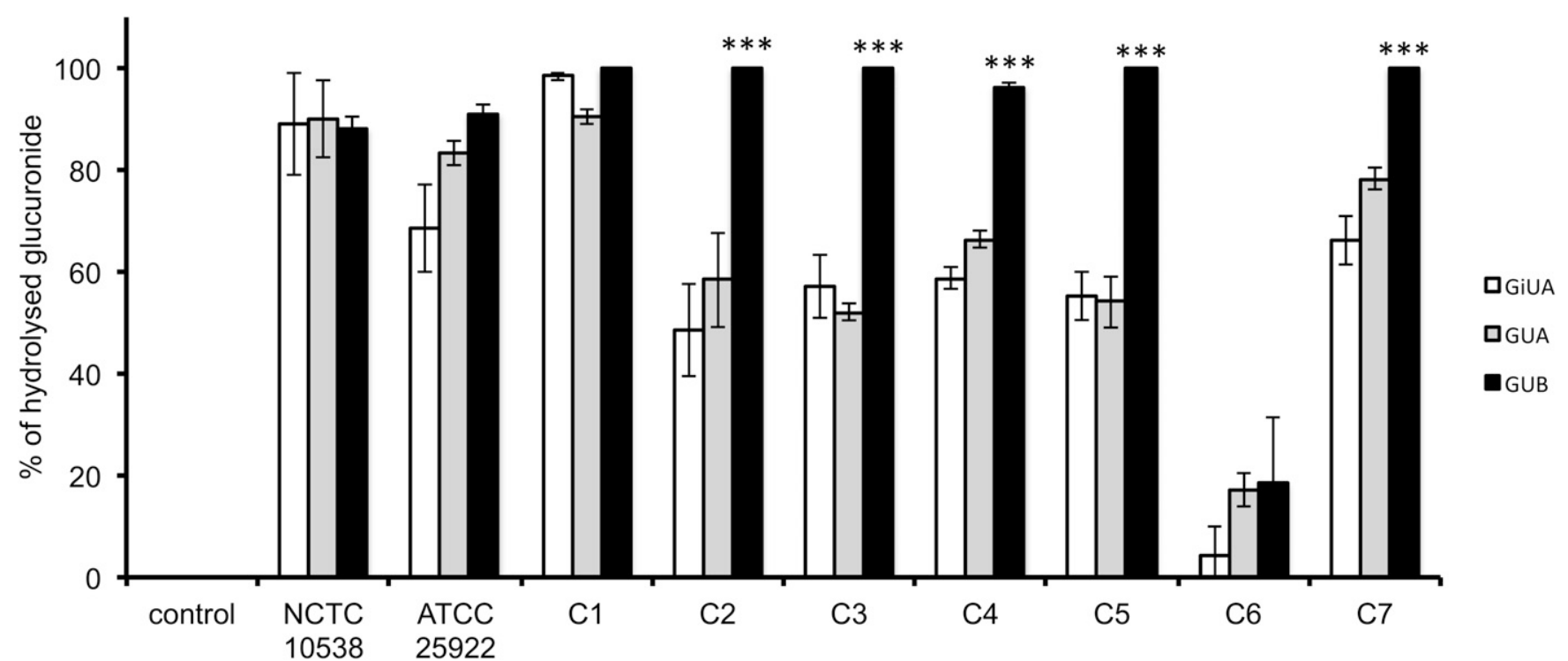

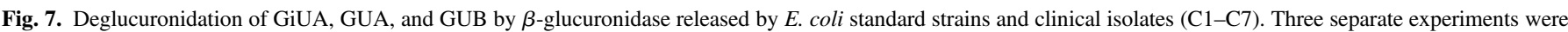

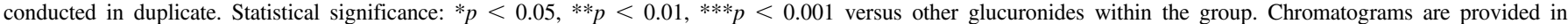
Supplemental Fig. S1. 
in $\mathrm{pH}$ decreases due to elevated lactic acid production that creates optimal conditions for $\beta$-glucuronidase activity (Warburg, 1956a,b; Palsson-McDermott and O'Neill, 2013). As a result, autophagy impairment leads to mitochondrial dysfunction that induces $\beta$-glucuronidase activity (Ishisaka et al., 2013), whereas damaged mitochondria, similar to bacteria, release formyl peptides that can signal using formyl peptide receptor-1 to trigger an immune response (Sun et al., 2016).

We have clearly shown that urolithin conjugates are cleaved by $\beta$-glucuronidase, which is released by neutrophils from azurophilic granules upon stimulation of formyl peptide receptor-1. The optimum $\mathrm{pH}$, obtained by acidification with lactic acid, was necessary for enzymatic cleavage, as it was not observed in either the environment of pure HBSS or that acidified to $\mathrm{pH}=3.5$. Previous studies conducted for luteolin glucuronide have revealed that its deconjugation is triggered by stimulated neutrophils. An increased ratio of aglycone/glucuronide was also observed in the plasma of LPS-treated rats (Shimoi et al., 2001). The $\beta$-glucuronidase released by macrophages was shown to be essential for activation of quercetin glucuronide conjugates. Its enzymatic activity, which requires acidic $\mathrm{pH}$, was promoted by increased secretion of lactate in response to mitochondrial dysfunction (Kawai, 2014).

The role of selective deconjugation within neoplastic tissues has attracted considerable attention. Since high levels of $\beta$-glucuronidase can be found in most solid tumors, a wide number of structurally diverse glucuronide prodrugs have been designed with the aim of enhancing the selectivity of cancer chemotherapy (Tranoy-Opalinski et al., 2014).

Although the highest concentrations of urolithin phase II metabolites were observed in urine, no studies have been conducted yet regarding the impact of these compounds on ailments associated with urinary tract disorders. Uropathogenic E. coli is the main cause of urinary tract infections, which produces significant amounts of $\beta$-glucuronidase (Vinacur et al., 1974; Helander and Dahl, 2005; McLellan and Hunstad, 2016). We have shown that standard strains of $E$. coli, as well as clinical isolates from patients with urinary tract infections, were capable of urolithin glucuronide cleavage. Previous studies conducted on the phase II metabolites of hydroquinone have shown that $E$. coli cleaved hydroquinone conjugates and liberated toxic-free hydroquinone, which is known to possess antibacterial activity (Siegers et al., 2003). To date, no studies regarding the urolithin effects of urinary tract disorders, $E$. coli viability, and virulence factors have been conducted. Nevertheless, deconjugation of urolithin glucuronides by $E$. coli $\beta$-glucuronidase revealed here indicates the need for examination of both conjugated and nonconjugated urolithins in future studies of the influence of orally administered ET-containing natural products on processes associated with urinary tract infections.

The dominance of urolithins of plasma, tissues, and urine glucuronide conjugates is potent, as they release their aglycones directly in inflamed and neoplastic tissues due to enhanced $\beta$-glucuronidase activity. The release of free aglycones is also possible within urinary tracts infected with $\beta$-glucuronidase-producing $E$. coli strains. Strong biologic activities, such as the antineoplastic and anti-inflammatory activities already described for nonconjugated urolithins (Tomás-Barberán et al., 2017), make intensive detoxification through phase II metabolism plausible. This process could be responsible for prevention of the impact of urolithins on biochemical processes throughout the entire organism. Thus, the conjugation of these pharmacologically active metabolites can be seen as a beneficial process, especially considering the formation of these compounds by gut microbiota from ETs that are widely distributed in various food products. It can be hypothesized that the selective activation of urolithin glucuronides by $\beta$-glucuronidase present at high concentrations at the inflammation site and in the microenvironments of most solid tumors could only locally increase the concentration of bioactive urolithin aglycones. This action can prevent their impact on cellular processes within healthy tissues. To fully support the stated hypothesis, the observed deconjugation should be further confirmed in in vivo studies. However, the presented results provide new insights into understanding urolithin disposition.

\section{Authorship Contributions}

Participated in research design: Piwowarski, Stefańska, Kiss.

Conducted experiments: Piwowarski, Stanisławska.

Contributed new reagents or analytic tools: Granica, Piwowarski.

Performed data analysis: Piwowarski.

Wrote or contributed to the writing of the manuscript: Piwowarski, Stanisławska.

\section{References}

Adams LS, Zhang Y, Seeram NP, Heber D, and Chen S (2010) Pomegranate ellagitannin-derived compounds exhibit antiproliferative and antiaromatase activity in breast cancer cells in vitro. Cancer Prev Res (Phila) 3:108-113.

Chang GW, Brill J, and Lum R (1989) Proportion of beta-D-glucuronidase-negative Escherichic coli in human fecal samples. Appl Environ Microbiol 55:335-339.

Dellafiora L, Mena P, Cozzini P, Brighenti F, and Del Rio D (2013) Modelling the possible bioactivity of ellagitannin-derived metabolites. In silico tools to evaluate their potential xenoestrogenic behavior. Food Funct 4:1442-1451.

Espín JC, Larrosa M, García-Conesa MT, and Tomás-Barberán F (2013) Biological significance of urolithins, the gut microbial ellagic Acid-derived metabolites: the evidence so far. Evid Based Complement Alternat Med 2013:270418.

García-Villalba R, Espín JC, and Tomás-Barberán FA (2016) Chromatographic and spectroscopic characterization of urolithins for their determination in biological samples after the intake of foods containing ellagitannins and ellagic acid. J Chromatogr A 1428:162-175.

Giménez-Bastida JA, González-Sarrías A, Larrosa M, Tomás-Barberán F, Espín JC, and GarcíaConesa MT (2012) Ellagitannin metabolites, urolithin A glucuronide and its aglycone urolithin A, ameliorate TNF- $\alpha$-induced inflammation and associated molecular markers in human aortic endothelial cells. Mol Nutr Food Res 56:784-796.

González-Barrio R, Borges G, Mullen W, and Crozier A (2010) Bioavailability of anthocyanins and ellagitannins following consumption of raspberries by healthy humans and subjects with an ileostomy. J Agric Food Chem 58:3933-3939.

González-Barrio R, Truchado P, Ito H, Espín JC, and Tomás-Barberán FA (2011) UV and MS identification of Urolithins and Nasutins, the bioavailable metabolites of ellagitannins and ellagic acid in different mammals. J Agric Food Chem 59:1152-1162.

González-Sarrías A, Espín JC, Tomás-Barberán FA, and García-Conesa MT (2009) Gene expression, cell cycle arrest and MAPK signalling regulation in Caco- 2 cells exposed to ellagic acid and its metabolites, urolithins. Mol Nutr Food Res 53:686-698.

González-Sarrías A, Giménez-Bastida JA, García-Conesa MT, Gómez-Sánchez MB, García-Talavera NV, Gil-Izquierdo A, Sánchez-Alvarez C, Fontana-Compiano LO, Morga-Egea JP, Pastor-Quirante FA, et al. (2010) Occurrence of urolithins, gut microbiota ellagic acid metabolites and proliferation markers expression response in the human prostate gland upon consumption of walnuts and pomegranate juice. Mol Nutr Food Res 54:311-322.

González-Sarrías A, Giménez-Bastida JA, Núñez-Sánchez MA, Larrosa M, García-Conesa MT, Tomás-Barberán FA, and Espín JC (2014) Phase-II metabolism limits the antiproliferative activity of urolithins in human colon cancer cells. Eur J Nutr 53:853-864.

González-Sarrías A, Miguel V, Merino G, Lucas R, Morales JC, Tomás-Barberán F, Alvarez AI, and Espín JC (2013) The gut microbiota ellagic acid-derived metabolite urolithin A and its sulfate conjugate are substrates for the drug efflux transporter breast cancer resistance protein (ABCG2/BCRP). J Agric Food Chem 61:4352-4359.

González-Sarrías A, Núñez-Sánchez MÁ, García-Villalba R, Tomás-Barberán FA, and Espín JC (2017) Antiproliferative activity of the ellagic acid-derived gut microbiota isourolithin A and comparison with its urolithin A isomer: the role of cell metabolism. Eur J Nutr 56:831-841.

González-Sarrías A, Núñez-Sánchez MA, Tomé-Carneiro J, Tomás-Barberán FA, García-Conesa MT, and Espín JC (2016) Comprehensive characterization of the effects of ellagic acid and urolithins on colorectal cancer and key-associated molecular hallmarks: MicroRNA cell specific induction of CDKN1A (p21) as a common mechanism involved. Mol Nutr Food Res 60: 701-716.

Helander A and Dahl H (2005) Urinary tract infection: a risk factor for false-negative urinary ethy glucuronide but not ethyl sulfate in the detection of recent alcohol consumption. Clin Chem $\mathbf{5 1}$ $1728-1730$.

Ho KJ (1985) Human beta-glucuronidase. Studies on the effects of $\mathrm{pH}$ and bile acids in regard to its role in the pathogenesis of cholelithiasis. Biochim Biophys Acta 827:197-206.

Ishisaka A, Kawabata K, Miki S, Shiba Y, Minekawa S, Nishikawa T, Mukai R, Terao J, and Kawai Y (2013) Mitochondrial dysfunction leads to deconjugation of quercetin glucuronides in inflammatory macrophages. PLoS One 8:e80843.

Kawai Y (2014) $\beta$-Glucuronidase activity and mitochondrial dysfunction: the sites where flavonoid glucuronides act as anti-inflammatory agents. J Clin Biochem Nutr 54:145-150.

Kieran T and Del Rio D (2015) Diet-Microbe Interactions in the Gut. Effects on human health and disease, Academic Press, London.

Klaassen CD and Cui JY (2015) Review: mechanisms of how the intestinal microbiota alters the effects of drugs and bile acids. Drug Metab Dispos 43:1505-1521.

Larrosa M, García-Conesa MT, Espín JC, and Tomás-Barberán FA (2010a) Ellagitannins, ellagic acid and vascular health. Mol Aspects Med 31:513-539.

Larrosa M, González-Sarrías A, García-Conesa MT, Tomás-Barberán FA, and Espín JC (2006) Urolithins, ellagic acid-derived metabolites produced by human colonic microflora, exhibit estrogenic and antiestrogenic activities. J Agric Food Chem 54:1611-1620.

Larrosa M, González-Sarrías A, Yáñez-Gascón MJ, Selma MV, Azorín-Ortuño M, Toti S, TomásBarberán F, Dolara P, and Espín JC (2010b) Anti-inflammatory properties of a pomegranate 
extract and its metabolite urolithin-A in a colitis rat model and the effect of colon inflammation on phenolic metabolism. $J$ Nutr Biochem 21:717-725.

Lucas R, Alcantara D, and Morales JC (2009) A concise synthesis of glucuronide metabolites of urolithin-B, resveratrol, and hydroxytyrosol. Carbohydr Res 344:1340-1346.

McLellan LK and Hunstad DA (2016) Urinary Tract Infection: Pathogenesis and Outlook. Trends Mol Med 22:946-957.

Mora-Cubillos X, Tulipani S, Garcia-Aloy M, Bulló M, Tinahones FJ, and Andres-Lacueva C (2015) Plasma metabolomic biomarkers of mixed nuts exposure inversely correlate with severity of metabolic syndrome. Mol Nutr Food Res 59:2480-2490.

Nuñez-Sánchez MA, García-Villalba R, Monedero-Saiz T, García-Talavera NV, Gómez-Sánchez MB, Sánchez-Álvarez C, García-Albert AM, Rodríguez-Gil FJ, Ruiz-Marín M, Pastor-Quirante FA, et al. (2014) Targeted metabolic profiling of pomegranate polyphenols and urolithins in plasma, urine and colon tissues from colorectal cancer patients. Mol Nutr Food Res 58: 1199-1211.

Palsson-McDermott EM and O'Neill LA (2013) The Warburg effect then and now: from cancer to inflammatory diseases. BioEssays 35:965-973.

Pearson AG, Kiefel MJ, Ferro V, and von Itzstein M (2005) Towards the synthesis of aryl glucuronides as potential heparanase probes. An interesting outcome in the glycosidation of glucuronic acid with 4-hydroxycinnamic acid. Carbohydr Res 340:2077-2085.

Piwowarski JP, Granica S, and Kiss AK (2014a) Influence of gut microbiota-derived ellagitannins' metabolites urolithins on pro-inflammatory activities of human neutrophils. Planta Med $\mathbf{8 0}$ 887-895.

Piwowarski JP, Granica S, Stefańska J, and Kiss AK (2016) Differences in metabolism of ellagitannins by human gut microbiota ex vivo cultures. J Nat Prod 79:3022-3030.

Piwowarski JP, Granica S, Zwierzyńska M, Stefańska J, Schopohl P, Melzig MF, and Kiss AK (2014b) Role of human gut microbiota metabolism in the anti-inflammatory effect of traditionally used ellagitannin-rich plant materials. J Ethnopharmacol 155:801-809.

Piwowarski JP and Kiss AK (2013) C-glucosidic ellagitannins from Lythri herba (European Pharmacopoeia): chromatographic profile and structure determination. Phytochem Anal 24 336-348.

Piwowarski JP, Kiss AK, Granica S, and Moeslinger T (2015) Urolithins, gut microbiota-derived metabolites of ellagitannins, inhibit LPS-induced inflammation in RAW 264.7 murine macrophages. Mol Nutr Food Res 59:2168-2177.

Ryu D, Mouchiroud L, Andreux PA, Katsyuba E, Moullan N, Nicolet-Dit-Félix AA, Williams EG, Jha P, Lo Sasso G, Huzard D, et al. (2016) Urolithin A induces mitophagy and prolongs lifespan in C. elegans and increases muscle function in rodents. Nat Med 22:879-888.

Sala R, Mena P, Savi M, Brighenti F, Crozier A, Miragoli M, Stilli D, and Del Rio D (2015) Urolithins at physiological concentrations affect the levels of pro-inflammatory cytokines and growth factor in cultured cardiac cells in hyperglucidic conditions. J Funct Foods 15:97-105.
Seeram NP, Aronson WJ, Zhang Y, Henning SM, Moro A, Lee RP, Sartippour M, Harris DM, Rettig M, Suchard MA, et al. (2007) Pomegranate ellagitannin-derived metabolites inhibit prostate cancer growth and localize to the mouse prostate gland. J Agric Food Chem $\mathbf{5 5}$ $7732-7737$.

Shimoi K, Saka N, Nozawa R, Sato M, Amano I, Nakayama T, and Kinae N (2001) Deglucuronidation of a flavonoid, luteolin monoglucuronide, during inflammation. Drug Metab Dispos 29:1521-1524.

Siegers C, Bodinet C, Ali SS, and Siegers CP (2003) Bacterial deconjugation of arbutin by Escherichia coli. Phytomedicine 10 (Suppl 4):58-60.

Sun N, Youle RJ, and Finkel T (2016) The Mitochondrial Basis of Aging. Mol Cell 61:654-666. Tomás-Barberán FA, García-Villalba R, González-Sarrías A, Selma MV, and Espín JC (2014) Ellagic acid metabolism by human gut microbiota: consistent observation of three urolithin phenotypes in intervention trials, independent of food source, age, and health status. J Agric Food Chem 62:6535-6538.

Tomás-Barberán FA, González-Sarrías A, García-Villalba R, Núñez-Sánchez MA, Selma MV, García-Conesa MT, and Espín JC (2017) Urolithins, the rescue of "old" metabolites to understand a "new" concept: Metabotypes as a nexus among phenolic metabolism, microbiota dysbiosis, and host health status. Mol Nutr Food Res 61.

Tranoy-Opalinski I, Legigan T, Barat R, Clarhaut J, Thomas M, Renoux B, and Papot S (2014) $\beta$-Glucuronidase-responsive prodrugs for selective cancer chemotherapy: an update. Eur J Med Chem 74:302-313.

van der Hooft JJ, de Vos RC, Mihaleva V, Bino RJ, Ridder L, de Roo N, Jacobs DM, van Duynhoven JP, and Vervoort J (2012) Structural elucidation and quantification of phenolic conjugates present in human urine after tea intake. Anal Chem 84:7263-7271.

Vinacur JC, Casellas JM, Rubi RA, and Oneto EP (1974) Serum anti-Escherichia coli antibodies and urinary beta-glucuronidase for the diagnosis and control of evolution of urinary infection during pregnancy. Am J Obstet Gynecol 120:812-816.

Warburg O (1956a) On respiratory impairment in cancer cells. Science 124:269-270.

Warburg O (1956b) On the origin of cancer cells. Science 123:309-314.

Yip LY and Chan EC (2015) Investigation of host-gut microbiota modulation of therapeutic outcome. Drug Metab Dispos 43:1619-1631.

Address correspondence to: Dr. Jakub Piwowarski, Department of Pharmacognosy and Molecular Basis of Phytotherapy, Medical University of Warsaw, Faculty of Pharmacy, Banacha 1, 02-097 Warsaw, Poland. E-mail: jakub.piwowarski@ wum.edu.pl 